\title{
Roles of Milk Fat Globule-Epidermal Growth Factor 8 in Intestinal Inflammation
}

\author{
Ryusaku Kusunoki $^{\mathrm{a}}$ Shunji Ishihara ${ }^{\mathrm{a}}$ Monowar Aziz $^{\mathrm{b}}$ Akihiko Oka \\ Yasumasa Tada ${ }^{a}$ Yoshikazu Kinoshita ${ }^{a}$ \\ ${ }^{a}$ Department of Internal Medicine II, Shimane University School of Medicine, Izumo, Japan; ${ }^{\mathrm{b} C e n t e r}$ for \\ Immunology and Inflammation, The Feinstein Institute for Medical Research, Department of Surgical Research, \\ North Shore University Hospital and Long Island Jewish Medical Center, Manhasset, N.Y., USA
}

\section{Key Words}

MFG-E8 • Intestinal inflammation • Apoptosis •

$\alpha_{v} \beta_{3}$-Integrin $\cdot$ Phosphatidylserine

\begin{abstract}
Milk fat globule-epidermal growth factor 8 (MFG-E8), a glycoprotein secreted from various cells, enhances engulfment of apoptotic cells by forming a link between phosphatidylserine on apoptotic cells and $\alpha_{v} \beta_{3}$-integrin on phagocytes. This process is essential for maintaining the host immune system under physiological conditions. Apart from this scavenging function, MFG-E8 also directly regulates a variety of cellular functions, such as attenuating inflammation and healing of injured tissues. Furthermore, recent studies have revealed that MFG-E8 has anti-inflammatory and regenerating roles during intestinal inflammation. This review highlights novel findings regarding the roles of MFG-E8 in intestinal pathophysiology as well as its therapeutic potential for gut inflammatory disorders.

Copyright ๑ 2012 S. Karger AG, Base
\end{abstract}

\section{Introduction}

Apoptotic cells are rapidly engulfed by phagocytes to avoid the release of numerous inflammatory mediators from dying cells. This function is essential for maintaining immune homeostasis and highly regulated by various unique molecular mechanisms. The glycoprotein milk fat globule-epidermal growth factor 8 (MFG-E8) was originally discovered as a mammalian milk fat globule membrane component. Later, it was shown that MFG-E8 binds to apoptotic cells and bridges them to phagocytes for accelerating engulfment [1]. Severe inflammatory and autoimmune consequences with abnormal homeostasis in MFG-E8 null mice are due to infiltration by apoptotic cells [2]. Recent studies have also revealed that MFG-E8 is functionally involved in the pathogenesis of sepsis, ischemia, atherosclerosis, and neurodegenerative disorders [3-6].

In addition to its scavenging function, MFG-E8 was shown to be effective in attenuating inflammation, by controlling epithelial integrity and healing of injured mucosa in the intestinal tract $[7,8]$. Those functions have been suggested to be dependent not only on enhanced clearance of apoptotic cells, but also on various novel molecular mechanisms. We recently reported that MFG-E8 attenuated in-

\section{KARGER \\ Fax +4161306 1234 E-Mail karger@karger.ch} www.karger.com
Shunji Ishihara, MD, $\mathrm{PhD}$

Department of Internal Medicine II, Shimane Medical University

89-1, Enya-cho, Izumo, Shimane 693-8501 (Japan)

Tel. +81 853202190

E-Mailsi360405@med.shimane-u.ac.jp 


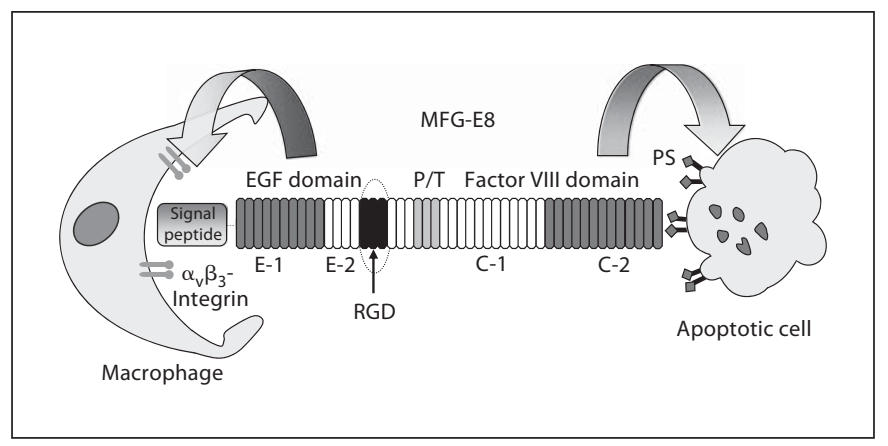

Fig. 1. Structure and noble functions of MFG-E8. The long form of murine MFG-E8 is a $64-\mathrm{kDa}$ glycoprotein, with two cysteinerich EGF domains at the $\mathrm{N}$-terminus and two discoidin-like domains resembling blood coagulation factor V/V111 at the Cterminal site. The nascent MFG-E8 peptide possesses a signal sequence that directs the protein to be secreted into the extracellular region. The most prominent function of MFG-E8 is to clear apoptotic cells by forming a link between PS on apoptotic cells and $\alpha_{\mathrm{v}} \beta_{3}$-integrin on phagocytes.

testinal inflammation in murine experimental colitis by modulating $\alpha_{v} \beta_{3}$-integrin signaling [9]. Since the intestinal tract is one of the major immune organs that contributes to maintaining normal tissue homeostasis, as well as regulating infections and inflammation, it is reasonable to speculate that MFG-E8 plays important roles in preserving intestinal functions. The aim of the present review is to highlight recent findings regarding the role of MFG-E8 in intestinal pathophysiology as well as its therapeutic potential for treatment of intestinal inflammation.

\section{Features of MFG-E8: Expression Profile and Its Regulation}

The MFG-E8 gene is located on chromosome 7 in mice and chromosome 15 in humans. In mouse tissues, MFGE8 is expressed as two different isoforms, termed short and long forms [1]. The structure of long-form MFG-E8 is shown in figure 1. MFG-E8 possesses two epidermal growth factor (EGF) repeats at the N-terminal and the blood coagulation factor V/VIII at the C-terminal end, while an arginine-glycine-aspartate (RGD) motif is contained in the second EGF repeat. MFG-E8 participates in phagocytosis of apoptotic cells by forming a link between phosphatidylserine (PS) on apoptotic cells and $\alpha_{v} \beta_{3}$-integrin on phagocytes [1].

MFG-E8 expression was initially discovered in samples obtained from lactating mammary glands. However, recent reports revealed that MFG-E8 is also ubiquitously expressed in the brain, heart, lungs, intestines, liver, and kidneys under normal physiological conditions [10-12]. We examined MFG-E8 expression in different tissues of normal $\mathrm{BALB} / \mathrm{c}$ mice and observed high levels in the colon, spleen, lungs, and kidneys [9]. In each organ, MFGE8 is expressed in a variety of cell types, including mammary epithelial cells, macrophages, splenocytes, dendritic cells, fibroblasts, vascular smooth muscle cells, glail cells, and astrocytes [13-18].

In various tissues and cells, MFG-E8 expression is tightly regulated by several factors and stimuli. Prolactin (PRL), a growth hormone, as well as insulin and steroid hormones are potent stimulators of MFG-E8 expression in their target cells $[11,19,20]$. We recently investigated the effects of PRL on MFG-E8 expression in macrophages by evaluating its promoter function [21]. Following treatment with PRL, significant up-regulation of MFG-E8 was observed in macrophages, while its effect was mediated by the presence of a responsive element of the transcription factor $\mathrm{C} / \mathrm{EBP} \beta$ in the MFG-E8 promoter. In addition, hormone-related regulation of MFG-E8 production, fractalkine (a CX3C chemokine), peroxisome proliferator-activated receptor (PPAR) $-\delta$ ligand, and granulocyte macrophage colony-stimulating factor (GM-CSF) have also been reported to induce MFG-E8 expression [16, 22, 23]. These factors are up-regulated in sites of inflammation in organs, suggesting that MFG-E8 may play essential roles for attenuating inflammation and regenerating injured tissues.

In contrast to the above findings, LPS is known to down-regulate MFG-E8 expression in macrophages. Komura et al. [3] used LPS-induced septic mice and found that endotoxemia decreased the endogenous levels of MFG-E8 in serum and several organs. Their findings also indicated that LPS-induced down-regulation of MFG-E8 expression in macrophages is mediated via the Toll-like receptor 4 (TLR4)/CD14 pathways.

\section{MFG-E8 Expression in Intestinal Tissues with Normal and Pathophysiological Stress}

As in other tissues and organs, basal levels of MFG-E8 expression have been observed in different compartments of mice gut tissues, e.g. the stomach, and small and large intestines, while that expression level in the colon was shown to be relatively higher as compared to the stomach and small intestine [9]. Furthermore, an immunohistochemical study detected MFG-E8 expression in lamina propria mononuclear cells in mice colonic sections. 
On the other hand, altered MFG-E8 expression has been found during intestinal inflammation. We recently examined changes of MFG-E8 expression during dextran sulfate sodium (DSS)-induced colitis in mice [9]. In that model, MFG-E8 expression was dramatically reduced during the acute phase of the disease, while it gradually became elevated during the regeneration phase after DSS in water intake was stopped and finally returned to a normal level when the disease was abrogated. Similar time-course changes of MFG-E8 expression were also found in a trinitrobenzene sulfonic acid (TNBS)-induced colitis model [24]. In an experimental model of sepsis established by cecal ligation and puncture, MFG-E8 levels in small intestinal tissues were markedly decreased [7]. Moreover, severe injury and inflammation were induced in small intestines of mice after intestinal ischemia and reperfusion (I/R), which decreased MFG-E8 levels in the spleen and other affected tissues [25]. Thus, MFG-E8 expression is down-regulated during the acute and severe inflammatory phases of intestinal disorders.

Although the underlying mechanism of this decreased production of MFG-E8 has not been clearly revealed, the abundance of pro-inflammatory mediators and involvement of LPS/TLR4 signaling may play important roles. One recent speculation states that the increased expression of MFG-E8 in injured intestinal mucosa during the acute phase of DSS-induced colitis may be due to the extent of inflammation and/or variations in mouse strains [26]. Consistent with these findings, in the majority of stress-induced disease conditions, e.g. renal I/R, alcoholintoxicated septic animals, and human atherosclerosis plaques, MFG-E8 expression has been found to be abruptly decreased $[4,8,27]$.

\section{Roles of MFG-E8 in Intestinal Inflammation}

\section{Anti-Inflammatory Effects}

Based on our findings of down-regulation of MFG-E8 during DSS-induced colitis, we treated mice with recombinant MFG-E8 (rMFG) and observed its beneficial effect to inhibit intestinal inflammation (fig. 2) [9]. In addition, an anti-inflammatory effect of rMFG was shown in a mouse model of I/R-induced intestinal injuries [25]. A recent study also revealed that DSS-induced colonic inflammation in MFG-E8 null mice is more severe than that in wild-type mice [26], indicating that MFG-E8 plays a crucial role in inhibiting intestinal inflammation (fig 2). In injured intestinal mucosa of MFG-E8 null mice, infiltration of apoptotic cells was not clearly evi-

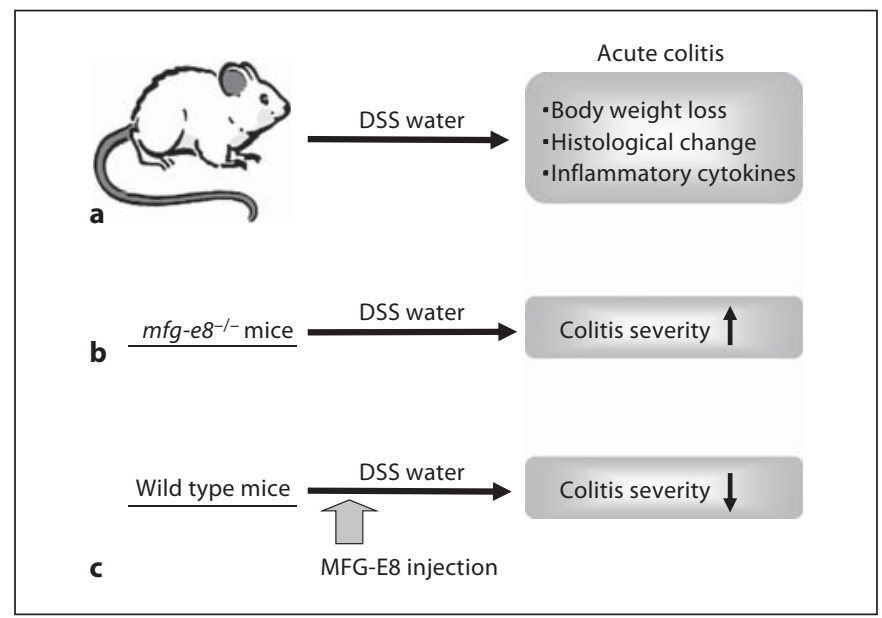

Fig. 2. Anti-inflammatory effects of MFG-E8 on DSS colitis. a Mice given DSS in drinking water showed clinical, histological, and inflammatory signs of colitis. b DSS-induced colonic inflammation in MFG-E8 null mice was more severe than that in wild-type mice. c rMFG-E8 effectively ameliorated the development of DSSinduced colitis by attenuating inflammation and disease status.

dent, suggesting that the protective role of MFG-E8 is not only due to efficient clearance of apoptotic cells, but also that this glycoprotein directly modulates innate immune functions [7]. Mice treated with rMFG-E8 at the onset of acute colitis showed significant down-regulation of the tissue contents of pro-inflammatory cytokines from inhibition of NF- $\mathrm{BB}$ activation [9]. During activation of the innate immune system, integrin signaling pathways are up-regulated, which then recognize potent ligands to boost the intracellular inflammatory cascade. Osteopontin (OPN) is an extracellular matrix phosphoprotein that contains the RGD domain, which is predominantly expressed in macrophages and induces the production of NF- $\kappa \mathrm{B}$-mediated inflammatory cytokines after binding to $\alpha_{\mathrm{v}} \beta_{3}$-integrin. We employed several in vitro experiments and observed that MFG-E8 reduced LPS-induced NF- $\kappa \mathrm{B}$ activation by blocking OPN binding, while it also modulated $\alpha_{\mathrm{v}} \beta_{3}$-integrin-dependent downstream signaling (fig. 3). After OPN binding, activation of $\alpha_{\mathrm{v}} \beta_{3}$-integrin also results in recruitment of phosphorylated focal adhesion kinase (FAK), leading to NF- $\kappa \mathrm{B}$ activation. Moreover, stimulation with LPS increases phosphorylation of FAK to enhance binding of exogenous OPN to $\alpha_{v} \beta_{3}$-integrin. By targeting this pathway, MFG-E8 can reduce LPS-induced NF- $\kappa \mathrm{B}$ activation by blocking OPN binding, as well as modulation of $\alpha_{\mathrm{v}} \beta_{3^{-}}$ integrin-dependent and FAK-mediated downstream sig- 


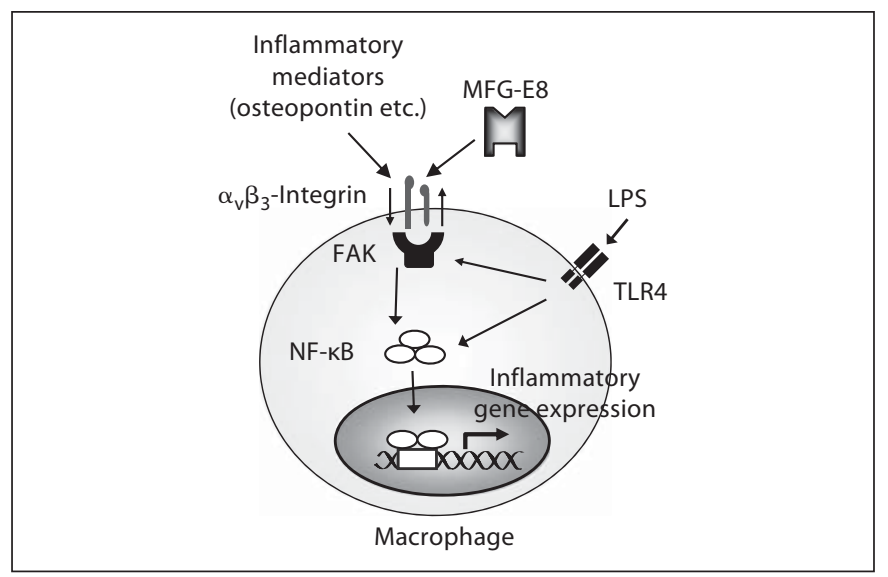

Fig. 3. Anti-inflammatory mediation roles of MFG-E8. LPS activates an innate immune response in macrophages via TLR4 signaling, which augments NF- $\kappa \mathrm{B}$-mediated production of pro-inflammatory cytokines. LPS also up-regulates $\alpha_{v} \beta_{3}$-integrin and generates inside-out signaling to promote the binding of several inflammatory mediators, e.g. OPN, which activates $\alpha_{v} \beta_{3}$-integrinmediated outside-in signaling to further augment the downstream pathways. After OPN binding, activation of $\alpha_{\mathrm{v}} \beta_{3}$-integrin also results in recruitment of phosphorylated FAK, leading to NF- $\mathrm{B}$ activation. Moreover, stimulation with LPS increases phosphorylation of FAK to enhance binding of exogenous OPN to $\alpha_{v} \beta_{3}$-integrin. By targeting this pathway, MFG-E8 can reduce LPS-induced NF- $\kappa$ activation by blocking OPN binding, as well as modulation of $\alpha_{\mathrm{v}} \beta_{3-}$ integrin-dependent and FAK-mediated downstream signaling.

naling (fig. 3). Similar to OPN, high-mobility group protein $\mathrm{B} 1$ (HMGB1) has the ability to directly bind to cell surface $\alpha_{\mathrm{v}} \beta_{3}$-integrin and induce tissue injury during colitis [28, 29]. MFG-E8 also competitively inhibits HMGB1 binding to $\alpha_{v} \beta_{3}$-integrin and may ameliorate HMGB1-mediated intestinal tissue injury.

Expressions of $\alpha_{\mathrm{v}} \beta_{3}$-integrins have been detected in intestinal epithelial cells (IECs) [24]. Also, MFG-E8 can inhibit TLR ligand-mediated production of pro-inflammatory cytokines by modulating NF- $\kappa \mathrm{B}$ activation in cultured IECs. This result is quite similar to that observed in an experiment that utilized macrophages. However, the detailed mechanisms of the anti-inflammatory effects of MFG-E8 on IECs remain unknown.

\section{Regeneration Role in Intestinal Injured Tissues}

$\mathrm{Bu}$ et al. [7] reported that MFG-E8 plays a crucial role in tissue regeneration during the healing process of injured colonic mucosa. They investigated whether MFGE8 stimulates IEC migration in an in vitro wound-healing model and observed that treatment with rMFG-E8 promoted the migration of IECs by activating intracellu- lar protein kinase $\mathrm{C}$ (PKC). In addition, administration of rMFG-E8 to experimental septic mice accelerated mucosal healing by binding to the transiently exposed PS receptor of the injured IECs. In DSS- and TNBS-mediated mice colitis models, increased levels of colonic MFGE8 were detected during the regenerating phase of colitis $[9,24]$, which may contribute to healing of injured colonic mucosa by promoting IEC migration. On the other hand, angiogenesis is also a crucial event for intestinal tissue regeneration. MFG-E8 binds to $\alpha_{v} \beta_{3}$-integrin on endothelial cells and accelerates vascular endothelial growth factor-induced angiogenesis under physiological and pathological conditions [30], which may contribute to colonic tissue regeneration during inflammation.

\section{Future Perspective}

The crucial roles of MFG-E8 have been delineated in several animal models as well as knock-out mice studies that mimicked human intestinal disorders. Notably, MFGE8 has been shown to have both anti-inflammatory and regenerating roles during colitis. However, most of those findings were obtained in experiments that used acute and severe intestinal inflammation models, and the precise roles of MFG-E8 in chronic gut immune disorders remain largely unknown. Moreover, there have been no studies of the expression and functions of MFG-E8 in human intestinal mucosa. On the other hand, recent findings have revealed direct roles of MFG-E8 in innate immune functions by activating regulatory $\mathrm{T}$ cells, and subsequent production of IL-10 and transforming growth factor- $\beta$, which may further promote immunoregulatory functions within the tissue microenvironment $[31,32]$. Collectively, these results will provide direction for future investigations of MFG-E8 administration for ameliorating gut inflammatory disorders, including inflammatory bowel diseases.

\section{Conclusion}

In this review, findings regarding the various roles of MFG-E8 in intestinal tissues are presented, indicating the glycoprotein to be an essential factor for maintaining intestinal homeostasis.

\section{Disclosure Statement}

The authors declare that no financial or other conflicts of interest exist in relation to the content of the article. 


\section{References}

1 Hanayama R, Tanaka M, Miwa K, Shinohara A, Iwamatsu A, Nagata S: Identification of a factor that links apoptotic cells to phagocytes. Nature 2002;417:182-187.

-2 Hanayama R, Tanaka M, Miyasaka K, Aozasa K, Koike M, Uchiyama Y, Nagata S: Autoimmune disease and impaired uptake of apoptotic cells in MFG-E8-deficient mice. Science 2004;304:1147-1150.

3 Komura H, Miksa M, Wu R, Goyert SM, Wang P: Milk fat globule epidermal growth factor-factor VIII is down-regulated in sepsis via the lipopolysaccharide-CD14 pathway. J Immunol 2009; 182:581-587.

4 Ait-Oufella H, Kinugawa K, Zoll J, Simon T, Boddaert J, Heeneman S, Blanc-Brude O, Barateau V, Potteaux S, Merval R, Esposito B, Teissier E, Daemen MJ, Lesèche G, Boulanger C, Tedgui A, Mallat Z: Lactadherin deficiency leads to apoptotic cell accumulation and accelerated atherosclerosis in mice. Circulation 2007;115:2168-2177.

$\checkmark 5$ Thorp E, Tabas I: Mechanisms and consequences of efferocytosis in advanced atherosclerosis. J Leukoc Biol 2009;86:1089-1095.

-6 Fuller AD, Van Eldik LJ: MFG-E8 regulates microglial phagocytosis of apoptotic neurons. J Neuroimmune Pharmacol 2008;3: 246-256.

-7 Bu HF, Zuo XL, Wang X, Ensslin MA, Koti V, Hsueh W, Raymond AS, Shur BD, Tan XD: Milk fat globule-EGF factor 8/lactadherin plays a crucial role in maintenance and repair of murine intestinal epithelium. J Clin Invest 2007;117:3673-3683.

8 Matsuda A, Jacob A, Wu R, Zhou M, Nicastro JM, Coppa GF, Wang P: Milk fat globule-EGF factor VIII in sepsis and ischemiareperfusion injury. Mol Med 2010;17:126133.

9 Aziz MM, Ishihara S, Mishima Y Oshima N, Moriyama I, Yuki T, Kadowaki Y, Rumi MA, Amano Y, Kinoshita Y: MFG-E8 attenuates intestinal inflammation in murine experimental colitis by modulating osteopontindependent $\alpha_{\mathrm{v}} \beta_{3}$ integrin signaling. J Immunol 2009; 182:7222-7232.

-10 Watanabe T, Totsuka R, Miyatani S, Kurata S, Sato S, Katoh I, Kobayashi S, Ikawa Y: Production of the long and short forms of MFGE8 by epidermal keratinocytes. Cell Tissue Res 2005;321:185-193.

-11 Aoki N, Ishii T, Ohira S, Yamaguchi Y, Negi M, Adachi T, Nakamura R, Matsuda T: Stage-specific expression of milk fat globule membrane glycoproteins in mouse mammary gland: comparison of MFG-E8, butyrophilin, and CD36 with a major milk protein, $\beta$-casein. Biochim Biophys Acta 1997;1334: 182-190.
12 Yamaguchi H, Fujimoto T, Nakamura S, Ohmura K, Mimori T, Matsuda F, Nagata S: Aberrant splicing of the milk fat globuleEGF factor 8 (MFG-E8) gene in human systemic lupus erythematosus. Eur J Immunol 2010;40:1778-1785.

13 Franchi A, Bocca S, Anderson S, Riggs R, Oehninger S: Expression of milk fat globule EGF-factor 8 (MFG-E8) mRNA and protein in the human endometrium and its regulation by prolactin. Mol Hum Reprod 2011;17: 360-371.

14 Miyasaka K, Hanayama R, Tanaka M, Nagata S: Expression of milk fat globule epidermal growth factor 8 in immature dendritic cells for engulfment of apoptotic cells. Eur J Immunol 2004;34:1414-1422.

15 Leonardi-Essmann F, Emig M, Kitamura Y, Spanagel R, Gebicke-Haerter PJ: Fractalkine-upregulated milk-fat globule EGF factor-8 protein in cultured rat microglia. J Neuroimmunol 2005;160:92-101.

16 Jinushi M, Nakazaki Y, Dougan M, Carrasco DR, Mihm M, Dranoff G: MFG-E8-mediated uptake of apoptotic cells by APCs links the pro- and anti-inflammatory activities of GM-CSF. J Clin Invest 2007;117:1902-1913.

17 Han X, Bolcato AL, Amar S: Identification of genes differentially expressed in cultured human osteoblasts versus human fibroblasts by DNA microarray analysis. Connect Tissue Res 2002;43:63-75.

18 Kranich J, Krautler NJ, Falsig J, Ballmer B, Li S, Hutter G, Schwarz P, Moos R, Julius C Miele G, Aguzzi A: Engulfment of cerebral apoptotic bodies controls the course of prion disease in a mouse strain-dependent manner. J Exp Med 2010;207:2271-2281.

19 Mather IH, Banghart LR, Lane WS: The major fat-globule membrane proteins, bovine components 15/16 and guinea-pig GP 55, are homologous to MGF-E8, a murine glycoprotein containing epidermal growth factorlike and factor V/VIII-like sequences. Biochem Mol Biol Int 1993;29:545-554.

-20 Oshima K, Aoki N, Negi M, Kishi M, Kitajima K, Matsuda T: Lactation-dependent expression of an mRNA splice variant with an exon for a multiply O-glycosylated domain of mouse milk fat globule glycoprotein MFG-E8. Biochem Biophys Res Commun 1999;254:522-528.

21 Aziz MM, Ishihara S, Rumi MA Mishima Y, Oshima N, Kadota C, Moriyama I, Li YY, Rahman FB, Otani A, Oka A, Ishimura N, Kadowaki Y, Amano Y, Kinoshita Y: Prolactin induces MFG-E8 production in macrophages via transcription factor $\mathrm{C} / \mathrm{EBP} \beta$-dependent pathway. Apoptosis 2008;13:609620.
22 Miksa M, Amin D, Wu R, Ravikumar TS, Wang P: Fractalkine-induced MFG-E8 leads to enhanced apoptotic cell clearance by macrophages. Mol Med 2007;13:553-560.

$>23$ Mukundan L, Odegaard JI, Morel CR, Heredia JE, Mwangi JW, Ricardo-Gonzalez RR, Goh YP, Eagle AR, Dunn SE, Awakuni JU, Nguyen KD, Steinman L, Michie SA, Chawla A: PPAR- $\delta$ senses and orchestrates clearance of apoptotic cells to promote tolerance. Nat Med 2009;15:1266-1272.

24 Otani A, Ishihara S, Aziz MM, Kusunoki R, Tada Y, Oka A, Moriyama I, Yuki T, Amano $\mathrm{Y}$, Kinoshita Y, Intrarectal administration of MFG-E8 protein ameliorates murine experimental colitis by inhibiting Src-mediated $\mathrm{NF}-\kappa \mathrm{B}$ activation in intestinal epithelial cells. Gastroenterology 2009;136:A701.

25 Cui T, Miksa M, Wu R, Komura H, Zhou M, Dong W, Wang Z, Higuchi S, Chaung W, Blau SA, Marini CP, Ravikumar TS, Wang P: Milk fat globule epidermal growth factor 8 attenuates acute lung injury in mice after intestinal ischemia and reperfusion. Am J Respir Crit Care Med 2010;181:238-246.

26 Chogle A, Bu HF, Wang X, Brown JB, Chou PM, Tan XD: Milk fat globule-EGF factor 8 is a critical protein for healing of dextran sodium sulfate-induced acute colitis in mice. Mol Med 2011;17:502-507.

$>27$ Wu R, Chaung WW, Zhou M, Ji Y, Dong W, Wang Z, Qiang X, Wang P: Milk fat globule EGF factor 8 attenuates sepsis-induced apoptosis and organ injury in alcohol-intoxicated rats. Alcohol Clin Exp Res 2010;34: 1625-1633

28 Yang H, Tracey KJ: Targeting HMGB1 in inflammation. Biochim Biophys Acta 2010; 1799:149-156.

29 Lotze MT, Tracey KJ. High-mobility group box 1 protein (HMGB1): nuclear weapon in the immune arsenal. Nat Rev Immunol 2005; 5:331-342.

30 Silvestre JS, Théry C, Hamard G, Boddaert J, Aguilar B, Delcayre A, Houbron C, Tamarat $\mathrm{R}$, Blanc-Brude O, Heeneman S, Clergue M, Duriez M, Merval R, Lévy B, Tedgui A, Amigorena S, Mallat Z: Lactadherin promotes VEGF-dependent neovascularization. Nat Med 2005;11:499-506.

31 Zhou YJ, Gao J, Yang HM, Yuan XL, Chen TX, He ZJ: The role of the lactadherin in promoting intestinal DCs development in vivo and vitro. Clin Dev Immunol 2010;2010: 357541.

32 Asano K, Miwa M, Miwa K, Hanayama R, Nagase H, Nagata S, Tanaka M: Masking of phosphatidylserine inhibits apoptotic cell engulfment and induces autoantibody production in mice. J Exp Med 2004;200:459467. 